\title{
Structure and luminescence of intrinsic localized states in sodium silicate glasses
}

\author{
Konstantinos Konstantinou, ${ }^{*}$ Dorothy M. Duffy, and Alexander L. Shluger \\ Department of Physics and Astronomy, University College London, Gower Street, London, WC1E 6BT, United Kingdom
}

(Received 21 July 2016; revised manuscript received 14 October 2016; published 16 November 2016)

\begin{abstract}
Sodium silicate glasses exhibit a characteristic luminescence with a maximum at about $3.4 \mathrm{eV}$, which is thought to be determined by optical excitation of local glass structures, called L centers. To investigate the atomic and electronic structures of these centers, we calculated the electronic properties of the ground and excited states of a sodium silicate glass using classical and $a b$ initio methods. Classical molecular dynamics was used to generate glass models of $\mathrm{Na}_{2} \mathrm{O}-3 \mathrm{SiO}_{2}$ molar composition, and the density functional theory (DFT), with hybrid functionals, was used to identify and characterize the geometric and electronic structures of L centers. The ground and excited $\mathrm{L}^{*}$ center states are studied, and their calculated excitation and luminescence transition energies are in good agreement with experimental data. The results confirm that the lowest triplet excited states in sodium silicate glass are associated with small clusters of $\mathrm{Na}$ ions and nonbridging oxygen atoms. These clusters serve as structural precursors for the localization of the excited states, and the broad distribution of the luminescence energies is correlated with the short-range order of the Na cations. The atomic and electronic structures of the electron $\mathrm{E}_{1}^{-}$and hole $\mathrm{H}_{1}^{+}$centers are also studied. These results provide a more detailed insight into the atomistic structure of localized states in these important glasses.
\end{abstract}

DOI: 10.1103/PhysRevB.94.174202

\section{INTRODUCTION}

Alkali silicate glasses of various compositions are among the most widely used materials in contemporary optics $[1,2]$. Theoretical understanding of their structure and optical properties is important fundamentally as they represent a wellstudied example of amorphous materials where theoretical models can be compared with the experimental data (see, e.g., Refs. [2-4]). Photoluminescence is a particularly useful and sensitive tool for studying the electronic energy states in glasses with short-range order [5,6]. Sodium silicate glasses are known to luminesce under ultraviolet and $\mathrm{x}$-ray irradiation in a wide range of temperatures with a maximum luminescence energy of $3.4 \mathrm{eV}$ [7]. Trukhin et al. [8] have measured the excitation spectra, the kinetics, and the degree of polarization of luminescence in high-purity sodium silicate glasses under excitation with ultraviolet light with photon energies exceeding $5.5 \mathrm{eV}$ [see Fig. 1(a)]. They concluded that the luminescence is caused by the intrinsic anisotropic structural motif in the glass, labeled L center, which is also responsible for the fundamental absorption edge of high-purity silicate glasses. This luminescence can be caused also by the decay of unstable color centers as a result of thermostimulated processes [7] or tunneling recombination of electron and hole centers $[8,9]$.

The first model of the energetic structure and electronic processes in sodium silicate glasses was proposed by Mackey et al. [7]. It assumed that the top of the valence band of the glass is determined by the electron states of nonbridging oxygen atoms, whereas the sodium ion states form the bottom of the conduction band. According to this model, x-ray irradiation produces intrinsic electron and hole color centers, and the subsequent luminescence is caused by the electron recombination with the hole center. They also established that the electron centers can be easily bleached by illuminating the glass with light corresponding to their absorption band

*konstantinos.konstantinou.13@ucl.ac.uk
(1-3 eV), but the hole centers are more stable and cannot be bleached by illuminating in their absorption band. Similar results have been obtained in $\mathrm{Na}$-doped $\mathrm{SiO}_{2}$ glass samples [10].

Mott and Davis [3,11] proposed a model based on Siegel's data [10] for the absorption spectra of $\mathrm{Na}$-doped $\mathrm{SiO}_{2}$ glass. It assumes that a complex of nonbridging oxygen and sodium ions produces donor levels in the $\mathrm{SiO}_{2}$ gap, $2.5 \mathrm{eV}$ above the valence band. An excitation of sodium silicate glass by photons whose energy lies in the fundamental absorption region leads to the creation of self-trapped excitons [3]. Trukhin et al. concluded that the long-wavelength intrinsic absorption tail of sodium silicate glasses and polarized luminescence, when the excitation takes place with polarized light, are caused by intrinsic L centers [8,12].

The simplest structural model of the $\mathrm{L}$ center is based on Mackey's proposition [7] that the lowest conduction band of the alkali silicates is due to the alkali s orbital. It can be viewed $[8,12]$ as a quasimolecular complex consisting of an $\mathrm{O}$ atom with single coordination to a $\mathrm{Si}$ atom and an alkali modifier located in the close environment, and can be represented as structural fragment $3 \mathrm{O}-\mathrm{Si}-\mathrm{O}^{-}-\mathrm{Na}^{+}$of the vitreous network [see Fig. 1(b)]. In this model, the low energy optical excitations are explained in terms of an electron transfer from a nonbridging oxygen (NBO) to a sodium ion. The ground state of the $\mathrm{L}$ center corresponds to ionic bonding between the NBO and the $\mathrm{Na}$ ion and is a singlet state, $\mathrm{S}_{0}$ [13]. The excited $\mathrm{L}^{*}$ center corresponds to covalent bonding between these atoms and has both singlet $S_{1}$ and a lower triplet $T_{1}$ state. The optical excitation results in the $S_{0} \rightarrow S_{1}$ transition, while the photoluminescence is attributed to the $T_{1} \rightarrow S_{0}$ transition, as highlighted in Fig. 1(a). These transitions correspond to the electron transfer between localized s orbitals of $\mathrm{Na}$ ion and $2 \mathrm{p}$ states of NBO atom.

The quasimolecular nature of the electronic transitions can be used to both characterize the short-range glass structure and to explain the sensitivity of the L center properties towards structural disorder [13]. The ionization of an electron from $\mathrm{Na}^{0}$ 

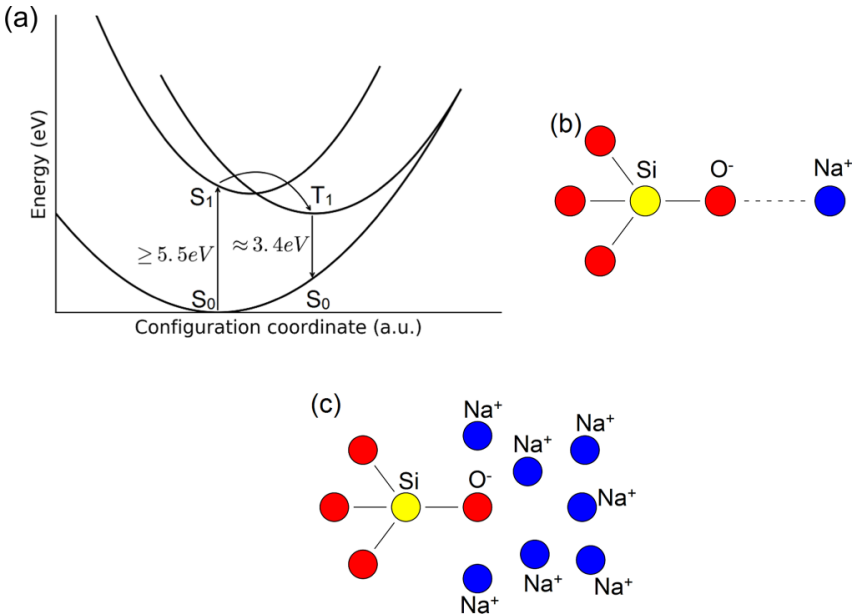

FIG. 1. (a) Schematic energy diagram corresponding to the ground and excited state for localized states in sodium silicate glass. The vertical transition from the relaxed ground state to the excited state corresponds to an energy $\geqslant 5.5 \mathrm{eV}$. After an intersystem crossing and geometry relaxation the system is in the triplet state and the luminescence corresponds to the vertical triplet-singlet transition. (b) The proposed structural model of the L center: a quasimolecular complex, which can be represented as a sodium cation in the vicinity of a nonbridging oxygen atom. (c) The extended L center model: a cluster of sodium cations located near an ordinary L center.

creates a hole center $\mathrm{H}_{1}^{+}$. Thus, the structure of the trapped-hole color center can be represented as $3 \mathrm{O}-\mathrm{Si}-\mathrm{O}^{0}-\mathrm{Na}^{+}$, with a strong $\mathrm{Na}$ ion displacement with respect to the NBO. An electron capture on the $\mathrm{L}$ center and the $\mathrm{Na}^{0}$ displacement from the NBO explains the $\mathrm{E}_{1}^{-}$center creation. Thus, the $\mathrm{E}_{1}^{-}$center's structure can be derived as $3 \mathrm{O}-\mathrm{Si}-\mathrm{O}^{-}-\mathrm{Na}^{0}$. The creation of $\mathrm{H}_{1}^{+}$ and $\mathrm{E}_{1}^{-}$centers at different distances explains the rich picture of luminescence kinetics under different pulse durations of excitation light, as well as the formation of color centers. An electron released thermally or optically from the $\mathrm{E}_{1}^{-}$center recombines with the $\mathrm{H}_{1}^{+}$center, and this leads to the restoration of the excited state of the center. Moreover, the recombination of $\mathrm{E}_{1}^{-}$and $\mathrm{H}_{1}^{+}$centers may occur by the electron tunneling mechanism.

The simple L center model qualitatively explains many effects in sodium silicate glasses, but it does not explain the disappearance of luminescence polarization at low temperatures [14,15]. This effect has been explained in terms of an extended L center model in a sodium silicate glass [13], which includes clusters of $\mathrm{Na}$ ions around the $3 \mathrm{O}-\mathrm{Si}-\mathrm{O}^{-}-\mathrm{Na}^{+}$ [see Fig. 1(c)]. Each sodium ion belongs to one or another $\mathrm{L}$ center. At low temperatures, an electron from an initial $\mathrm{Na}$ atom can transfer to a nearest ion and can be stabilized there.

A more recent analysis of the photoluminescence suggested that alkali silicate glasses have at least two types of $\mathrm{L}$ centers, $\mathrm{L}_{1}$ and $\mathrm{L}_{2}$, which are strongly dissimilar in their spectral and kinetic characteristics $[13,16,17]$. This was explained by the fact that the glass micro-structure contains fragments with different degrees of disorder in the environment of the L center. In addition, alkali silicate glasses have an inhomogeneous distribution of alkali cations within the structure, therefore the observed centers may occupy local regions that are depleted in alkali ions. Stationary exciton like states appear in optical absorption bands of $\mathrm{L}$ centers during the steady-state optical excitation. The radiative relaxation of these states is accompanied by the luminescence at $3.0-3.5 \mathrm{eV}$ and by electron emission $[13,18]$.

However, in spite of extensive experimental studies, no atomistic calculations of the $\mathrm{L}$ centers have been performed so far to investigate the proposed qualitative models and shed light on their atomistic structures. In this work, we used ab initio calculations to study the electronic and geometric structures of localized states in sodium silicate glass. The properties of the ground $\mathrm{L}$ and excited $\mathrm{L}^{*}$ center states and luminescence energies were calculated, together with the properties of the electron $\mathrm{E}_{1}^{-}$and hole $\mathrm{H}_{1}^{+}$centers. The results confirm that the lowest triplet excited states in sodium silicate glass are associated with clusters of $\mathrm{Na}$ ions and nonbridging oxygen atoms.

\section{COMPUTATIONAL DETAILS}

The calculations presented in this work make use of both classical force fields and density functional theory (DFT). Classical molecular dynamics (MD) simulations were used to generate sodium silicate glass models followed by periodic DFT calculations of the electronic structure and excitation and luminescence energies of these models.

\section{A. Classical simulations}

Twenty structures of sodium silicate glass of the composition $\mathrm{Na}_{2} \mathrm{O}-3 \mathrm{SiO}_{2}$ were generated using classical MD with periodic boundary conditions. The total number of atoms in each periodic cell is 192 with $\mathrm{Na}=26, \mathrm{Si}=51$, and $\mathrm{O}=115$. The atoms were placed randomly in a cubic simulation box, with a cell size calculated from the experimental density [19], with imposed constraints to avoid unphysically small interatomic distances.

A partial-charge rigid-ion pairwise potential developed by Pedone et al. [20] and employed in previous modeling studies of several silicate glasses [21-25] was used. The DL_POLY classic package [26] was used for the MD simulations. The velocity Verlet algorithm was applied to integrate the equations of motion with a timestep of $1 \mathrm{fs}$. The Coulomb interactions were calculated using the Ewald summation method [27] with a precision of $10^{-5}$. The cutoff for the short-range interactions was set to $6 \AA$.

The glass structures were generated using a standard melt-and-quench approach. The canonical ensemble (constant number of particles, volume and temperature or $N V T$ ) was applied to keep the density of the simulated glass close to the experimental value. The Berendsen thermostat [28], with a relaxation constant $0.1 \mathrm{ps}$, was used to control the temperature. The system was heated up gradually in steps of $100 \mathrm{~K}$ with a $60 \mathrm{ps} \mathrm{MD}$ run at each temperature. The molten structure was equilibrated at $6000 \mathrm{~K}$ using first the $N V T$ and then the NVE (microcanonical) ensembles with 400 ps MD run in total in order to ensure that the structure was well equilibrated at this temperature. The system was subsequently cooled down to $0 \mathrm{~K}$, using a uniform stepwise process, with temperature decrements of $50 \mathrm{~K}$ and $60 \mathrm{ps} N V T$ run at each 
temperature. This quench scheme corresponds to a cooling rate of approximately $0.8 \mathrm{~K} / \mathrm{ps}$.

The final structure was equilibrated for 200 ps using the constant-pressure and constant-temperature (NPT) ensemble so as to relax internal stresses. The Berendsen barostat and thermostat with relaxation constants 1.0 ps and $0.1 \mathrm{ps,}$ respectively, were used. A further 200 ps $N V E$ run was carried out, and the atomic trajectories were collected every 50 steps during the last $40 \mathrm{ps}$ of the final $N V E$ run. The structural data were averaged over these 800 configurations, and the accuracy of the model was validated by the comparison of the structure of the simulated glass with the experimental structural data.

Test simulations were run for different periodic cell sizes and cooling rates. The data present in the Supplemental Material (SI) [29] demonstrate that the glass structure does not depend significantly on the periodic cell size (see Table S1), which varied from 192 up to 12288 atoms, and on the cooling rate (see Table S2), which varied from 0.8 to $20 \mathrm{~K} / \mathrm{ps}$. We note that similar conclusions have been reached in a recent study by Tilocca [30]. Most of the calculations have been done at the cooling rate of $1 \mathrm{~K} / \mathrm{ps}$.

\section{B. Density functional theory calculations}

The output glass structures from the melt-and-quench calculations were used as input configurations, for each glass model, to further optimize their geometry using DFT implemented in the $\mathrm{CP} 2 \mathrm{~K}$ code and to calculate the electronic structures [31]. The CP2K code uses a Gaussian basis set with an auxiliary plane-wave basis set [32]. Employing a Gaussian basis set has the advantage of allowing one to use fast analytical integration schemes, developed in quantum mechanical methods, to calculate most of the Kohn-Sham matrix elements. The use of an auxiliary plane-wave basis set gives the opportunity to use fast Fourier transform algorithms for rapid convergence of the long-range Hartree terms. All atomic species were represented using a double- $\zeta$ valence polarized (DZVP) Gaussian basis set [33] in conjunction with the Goedecker-Teter-Hutter (GTH) pseudopotential [34]. The plane-wave cutoff was set to $1000 \mathrm{Ry}$. The nonlocal functional PBE0_TC_LRC was used in all calculations with a cutoff radius of $2.0 \AA$ [35]. The inclusion of the Hartree-Fock exchange provides an accurate description of the band gap and localized states of the $\mathrm{L}$ center in our sodium silicate glass models. The computational cost of nonlocal functional calculations can be reduced using the auxiliary density matrix method (ADMM) [36]. The density is mapped onto a much sparser Gaussian basis set containing less diffuse and fewer primitive Gaussian functions than the one employed in the rest of the calculation. This allows the Hartree-Fock exchange terms, whose computational expense scales to the fourth power of the number of basis function, to be calculated on a much smaller basis set than the rest of the calculation, which substantially reduces the computational time.

The singlet excited state of an L center is known to quickly convert into the triplet state [13]. The lowest triplet excited state in the relaxed glass structures was calculated using a ground-state DFT method by placing the system in the triplet state, which promotes an electron from the top of the valence band into the bottom of the conduction band. The geometry of the triplet state was found by minimizing the total energy of the system with respect to atomic positions. The excitation energy was calculated using the $\triangle$ SCF method $[37,38]$ as a difference between the total energies of the glass structure in the ground singlet and the lowest triplet state at the geometry of the ground singlet state. The luminescence energy was calculated using the $\triangle$ SCF method as the difference between the total energy of the fully relaxed triplet state and the total energy of the ground singlet state at the triplet state geometry.

The electron $\mathrm{E}_{1}^{-}$and hole $\mathrm{H}_{1}^{+}$centers were modeled by injecting an extra electron and hole, respectively, in the relaxed ground state glass structure and minimizing the energy with respect to the atomic coordinates. The BFGS optimizer was applied in all the geometry optimizations to minimize the forces on atoms to within $40 \mathrm{pN}\left(2.5 \times 10^{-2} \mathrm{eV}^{-1}\right)$.

\section{RESULTS OF CALCULATIONS}

\section{A. The glass structure}

To study the distribution of $\mathrm{L}$ center structures and excitation energies we used 20 periodic glass structure models of 192 atoms. The cell size was chosen on the basis of our recent studies of defects in amorphous silica, and it is adequate for accommodating the local relaxation of localized defects and calculating their electronic structures $[39,40]$. The number of these cells was determined by computer resources available for spin-polarized DFT calculations using a nonlocal functional, and the obtained statistics should be sufficient for qualitative conclusions.

In all 20 simulated glass structures obtained using classical $\mathrm{MD}, \mathrm{Si}$ is fully 4-coordinated by oxygen atoms with an average $\mathrm{Si}-\mathrm{O}$ interatomic distance of $1.62 \AA$. The typical values from the literature for the Si-O bond length are 1.58-1.64 $\AA$ for silicate glasses [19,41,42]. Wright et al. [43] have found an increase in $\mathrm{Si}-\mathrm{O}$ bond length for glasses with 22 and $31 \%$ mole $\mathrm{Na}_{2} \mathrm{O}$ concentrations. The maximum of $\mathrm{O}-\mathrm{Si}-\mathrm{O}$ bond angle distribution is located at $108.5^{\circ}$ and it is in good agreement with the value of the perfect tetrahedron $\left(109.5^{\circ}\right)$. Together with the fully 4-coordinated $\mathrm{Si}$ this means that $\mathrm{SiO}_{4}$ tetrahedra are only slightly distorted in the simulated glass structures. An average value of the $\mathrm{Si}-\mathrm{O}-\mathrm{Si}$ bond angle between the tetrahedron linkages is about $145.5^{\circ}$, whereas in pure $\mathrm{SiO}_{2}$ glass this angle is at $150^{\circ}$. Usually, the addition of impurities leads to decrease of the Si-O-Si bond angle in the silicate network. The average Na-O bond length is $2.37 \AA$, and the average oxygen coordination number around $\mathrm{Na}$ is 5.8. Pairwise radial distribution functions for the $\mathrm{Na}-\mathrm{O}$ and $\mathrm{Na}-\mathrm{Na}$ interatomic distances are shown in Fig. S1 in SI [29]. The distribution of $\mathrm{Na}$ ions with $0,1,2,3$, and 4 other $\mathrm{Na}$ ions in the nearest neighbor positions in the glass structure is shown in Fig. S2 in SI [29]. One can see that, although about $21 \%$ of the Na ions do not have other $\mathrm{Na}$ ions nearby, about $55 \%$ of the $\mathrm{Na}$ ions have 1 or $2 \mathrm{Na}$ ions in the nearest surroundings and the remaining $24 \% 3$ or even $4 \mathrm{Na}$ ions. These values agree with the results of both neutron diffraction [43] and EXAFS [44] studies as well as with previous modeling studies in sodium silicate glasses [19,21,41,42] and demonstrate the inhomogeneous distribution of $\mathrm{Na}$ ions inside the glass structure with $\mathrm{Na}$ ion clusters with a range of sizes. 


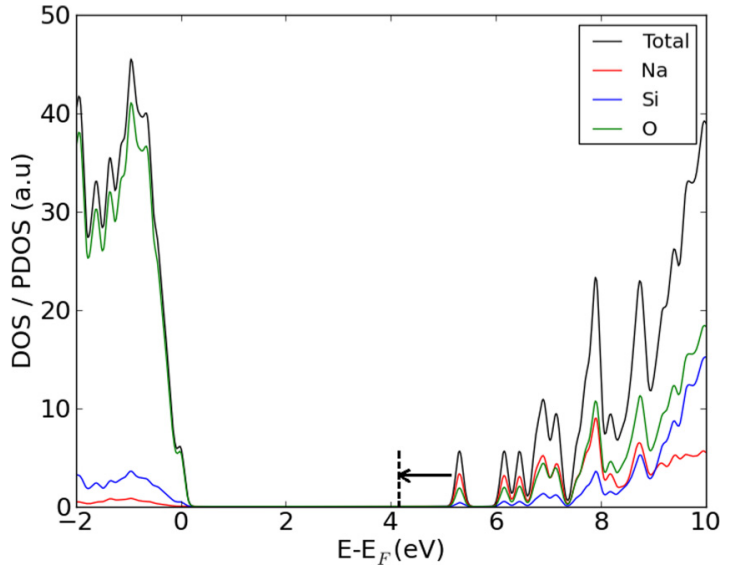

FIG. 2. Total and partial electronic density of states of a $\mathrm{Na}_{2} \mathrm{O}-3 \mathrm{SiO}_{2}$ glass model. The bottom of the conduction band is due to the $3 s \mathrm{Na}$ ion states, and the top of the valence band is due to the oxygen $2 p$ states. An extra electron will occupy the lowest unoccupied molecular orbital (LUMO), and this state will be shifted by $\approx 1.2 \mathrm{eV}$ towards the middle of the conduction band following the geometry relaxation, as indicated by the arrow.

The glass structures obtained using the classical force field were further optimized using DFT. The results shown in Table S3 in the SI [29] demonstrate that the changes to the geometric parameters of the glass structure following the geometry relaxation are insignificant, with interatomic distances remaining within $0.02 \AA$ of the original values. This demonstrates the quality of interatomic potentials [20] used in this work.

The electronic structure calculations show an average HOMO-LUMO Kohn-Sham band gap of $5.3 \mathrm{eV}$. The total and partial electronic density of states of one of the glass models is shown in Fig. 2. The bottom of the conduction band is due to the sodium ion states, whereas the electron states of nonbridging oxygens determine the top of the valence band. This is in good agreement with Mackey's [7] main assumption that the lowest conduction band of the alkali silicate glasses is due to the alkali s orbital, and the structural model of the L center was based on this proposition. The band gap is much lower than that of amorphous $\mathrm{SiO}_{2}(9 \mathrm{eV})$ due to the $\mathrm{Na}$ conduction band. It agrees well with the optical absorption edges of about $6 \mathrm{eV}$ for sodium silicate glasses reported by Mackey et al. [7] and Siegel [10]. Murray and Ching [45] also calculated the band gap at $4.6 \mathrm{eV}$ for $\mathrm{Na}_{2} \mathrm{O}-2 \mathrm{SiO}_{2}$ glass compositions. The band gap of the system would decrease with increasing the $\mathrm{Na}_{2} \mathrm{O}$ content.

\section{B. Excited state}

An average $5.6 \mathrm{eV}$ optical excitation energy obtained from the 20 glass models is in good agreement with the experimental studies $[8,12]$ where high-purity sodium silicate glasses were excited with ultraviolet light with photon energies exceeding $5.5 \mathrm{eV}$. As expected for a disordered system [3], the excited state is not fully delocalized in the periodic cell but is rather localized on several (typically 2 or 3 ) $\mathrm{Na}$ ions and 1 to 3 oxygen ions, forming the bottom of the conduction band and the top of the valence band, respectively (see Fig. 3).

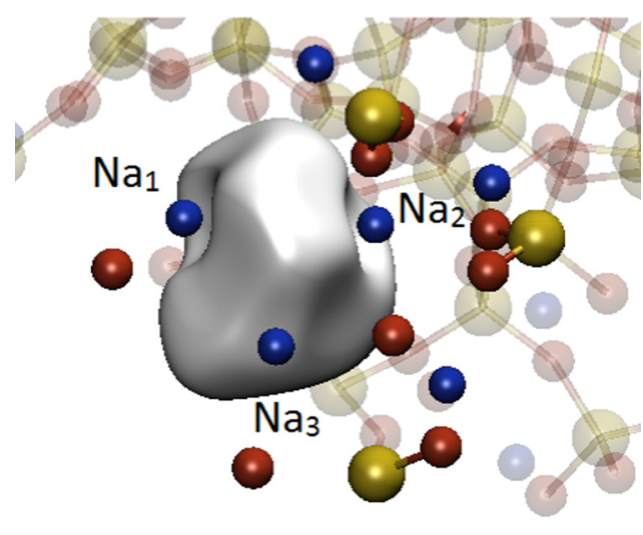

FIG. 3. Spin density distribution of the triplet excited state before relaxation in one of the glass models. The electron shows a preference to localization between three $\mathrm{Na}$ cations within the glass structure. $\mathrm{Na}$ ions are blue, $\mathrm{Si}$ are yellow, and $\mathrm{O}$ are red. The spin density isovalue is 0.002 .

The atomic structure exposed by this excited state is that of an $\mathrm{L}$ center. In the 20 structures studied here, the $\mathrm{L}$ center structure appears to be more complex than the simple $3 \mathrm{O}-\mathrm{Si}-\mathrm{O}^{-}-\mathrm{Na}^{+}$structure with several $\mathrm{Na}^{+}$ions and $\mathrm{O}^{-}$ions usually involved. We can qualitatively distinguish two types of $L$ center structures after vertical excitation shown in Fig. 4: type 1 , which have compact $\mathrm{Na}$ ion clusters consisting of three ions separated by about $3.6 \AA$, and type 2 , where the separation between $\mathrm{Na}$ ions is about $4.8 \AA$ on average. We note that, due to a relatively small number of $\mathrm{Na}$ ions in the periodic cell, our results may be prone to fluctuations and thus insufficient for describing all possible structures. Nevertheless, we treat these structures as representative for the purpose of this study.

The relaxed structure of the $\mathrm{L}^{*}$ center was determined by minimizing the total energy of the excited system with respect to the atomic coordinates. The typical spin density distribution of the relaxed triplet excited state is shown in Fig. 5. It is qualitatively similar to the one after the vertical excitation, but the character of ionic relaxation differs in different glass structures. Qualitatively, in type 1 structures we observe relatively small $(\leqslant 0.1 \AA)$ displacements of $\mathrm{Na}$ and $\mathrm{O}$ ions from their original sites, whereas in type 2 structures there are very large displacements, often approaching $1.0 \AA$, strongly
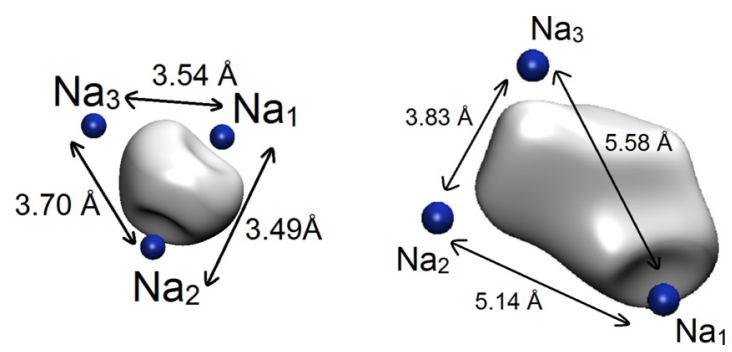

FIG. 4. Atomic structure and spin density distribution in the two types of L center structures. Type 1 (left panel) corresponds to a compact cluster of three $\mathrm{Na}$ ions with average $\mathrm{Na}-\mathrm{Na}$ interatomic distances of $\approx 3.6 \AA$, and type 2 (right panel) corresponds to a cluster of $3 \mathrm{Na}$ ions but with much larger separation between the cations. The isovalue of spin density is 0.002 . 


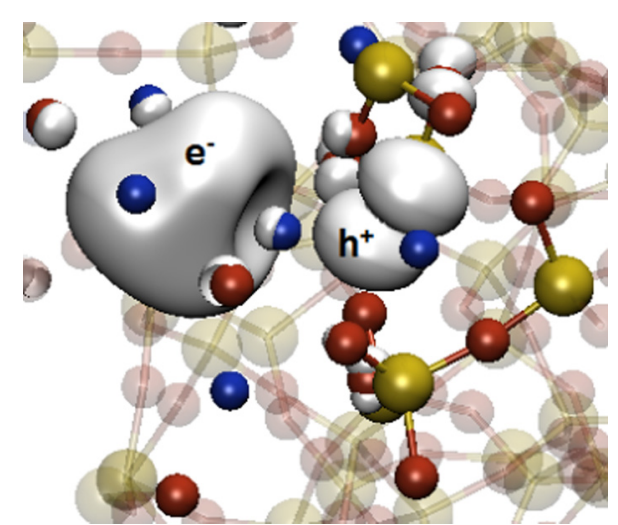

FIG. 5. Spin density distribution of the relaxed triplet excited state. The electron and hole components are indicated with $\mathrm{e}^{-}$and $\mathrm{h}^{+}$, respectively. The electron is localized around $3 \mathrm{Na}$ cations and the hole is localized on one nonbridging oxygen atom. Na ions are blue, $\mathrm{Si}$ are yellow, and $\mathrm{O}$ are red. The isovalue of spin density is equal to 0.002 .

affecting the luminescence energy. These excited states can be considered as relaxed triplet excitons in alkali silicate glasses [46].

\section{Luminescence energy}

The luminescence energies were calculated as the energy difference of the ground state (singlet) in the geometry of the relaxed triplet excited state and the relaxed triplet state. The calculated luminescence energies in our sodium silicate glass models are directly related to the two types of the excited state. In $60 \%$ of the glass samples (12 structures) where L centers have type 1 structures, the calculated luminescence energy is within the range of $2.7-3.3 \mathrm{eV}$, whereas the remaining eight type $2 \mathrm{~L}$ centers have luminescence energies in the range of $1.0-1.7 \mathrm{eV}$.

The calculated luminescence energies of the first group are in good agreement with the experimental values in sodium silicate glasses, which are known to exhibit a wide luminescence spectrum with the maximum at $3.4 \mathrm{eV}[7,8]$. In addition, the values of this range agree well with the luminescence spectra of sodium silicate glass with the same molar composition $\left(\mathrm{Na}_{2} \mathrm{O}-3 \mathrm{SiO}_{2}\right)$ reported by Zatsepin et al. [17]. These spectra, as well as those shown in Ref. [46], also exhibit red tails extending down to $2.0 \mathrm{eV}$.

\section{Atomistic relaxation in $L^{*}$ centers}

To investigate how the luminescence energies correlate with the changes in the geometry of the relaxed triplet excited state with respect of that of the relaxed ground state, we calculated the average atomic displacements for the three component species ( $\mathrm{Na}, \mathrm{O}$, and $\mathrm{Si}$ ) in each of the 20 glass models. These displacements and the luminescence energies are shown in Table I. One can clearly see that the lower luminescence energies correspond to larger average displacements of $\mathrm{Na}$ and $\mathrm{O}$ atoms in the relaxed $\mathrm{L}^{*}$ center. For the type $1 \mathrm{~L}^{*}$ center structures, having larger luminescence energies, the atomic displacements are on average considerably smaller.
TABLE I. Average displacement, $d$ (in $\AA$ ), between the geometry of the relaxed excited state and the geometry of the relaxed ground state for $\mathrm{Na}, \mathrm{O}$, and $\mathrm{Si}$ in the 20 glass models and the calculated luminescence energies $\mathrm{E}_{L}$ (in $\mathrm{eV}$ ).

\begin{tabular}{lcccc}
\hline \hline Glass & $\mathrm{E}_{L}$ & $d_{\mathrm{Na}}$ & $d_{\mathrm{O}}$ & $d_{\mathrm{Si}}$ \\
\hline 1 & 3.3 & 0.04 & 0.04 & 0.03 \\
2 & 3.2 & 0.09 & 0.05 & 0.03 \\
3 & 3.2 & 0.04 & 0.04 & 0.03 \\
4 & 3.2 & 0.06 & 0.04 & 0.02 \\
5 & 3.2 & 0.04 & 0.03 & 0.03 \\
6 & 3.1 & 0.04 & 0.05 & 0.04 \\
7 & 3.1 & 0.06 & 0.08 & 0.06 \\
8 & 3.0 & 0.05 & 0.03 & 0.03 \\
9 & 2.9 & 0.08 & 0.04 & 0.02 \\
10 & 2.9 & 0.08 & 0.06 & 0.04 \\
11 & 2.8 & 0.05 & 0.06 & 0.05 \\
12 & 2.7 & 0.07 & 0.07 & 0.05 \\
13 & 1.7 & 0.11 & 0.06 & 0.04 \\
14 & 1.7 & 0.25 & 0.12 & 0.09 \\
15 & 1.6 & 0.14 & 0.08 & 0.06 \\
16 & 1.6 & 0.13 & 0.07 & 0.05 \\
17 & 1.3 & 0.17 & 0.06 & 0.04 \\
18 & 1.2 & 0.10 & 0.07 & 0.05 \\
19 & 1.1 & 0.20 & 0.13 & 0.11 \\
20 & 1.0 & 0.19 & 0.10 & 0.07 \\
\hline \hline
\end{tabular}

To illustrate in more detail how the displacements of atoms within a particular structure are related to the electron and hole localization in the excited state, we present the results for the glass sample \#14 from Table I, which exhibits the largest average displacement for the $\mathrm{Na}$ atoms. The displacements of all the 192 atoms in the relaxed excited state with respect to their positions in the ground state are shown as a function of the distance of each atom from the center of mass of the $\mathrm{L}^{*}$ center structure in Fig. 6.

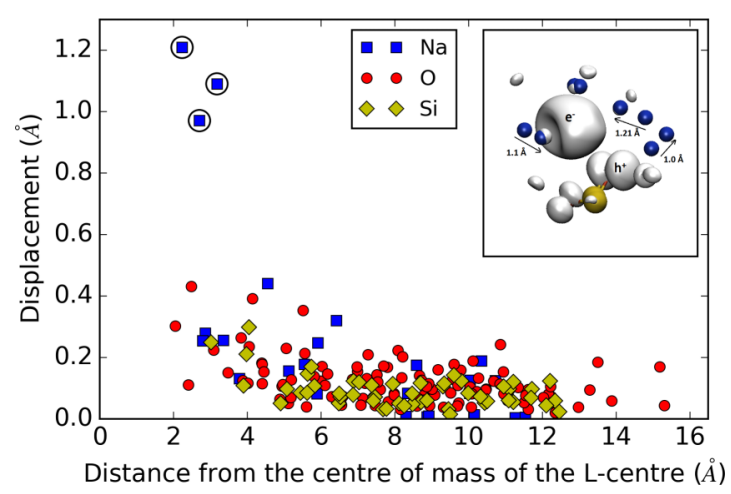

FIG. 6. Atomic displacements for $\mathrm{Na}, \mathrm{Si}$, and $\mathrm{O}$ atoms between the geometry of the relaxed ground state and the relaxed excited state in a type 2 excited state (glass \#14 in Table I) as a function of the distance of each atom from the center of mass of the L center. Inset: Spin density distribution (isovalue is 0.002 ) and atomic structure of the localized excited state. Encircled are the displacements of the three $\mathrm{Na}$ ions most involved in the exciton $\left(\mathrm{e}^{-}-\mathrm{h}^{+}\right)$localization. The direction of the arrows corresponds to the direction of the motion from the relaxed ground state to the relaxed excited state. 


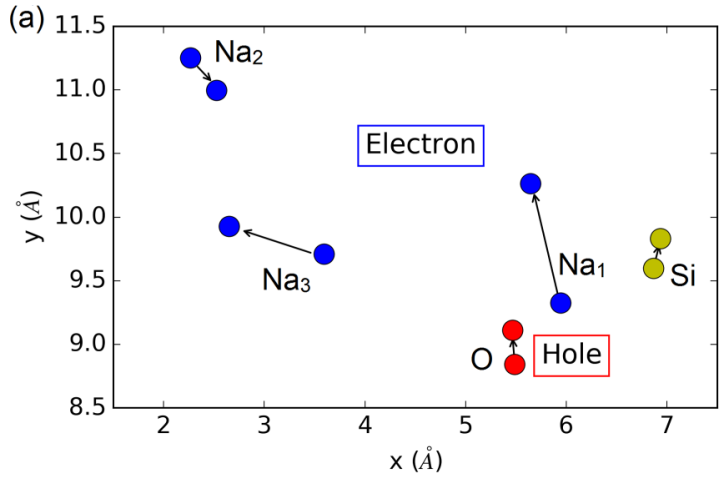

(b)

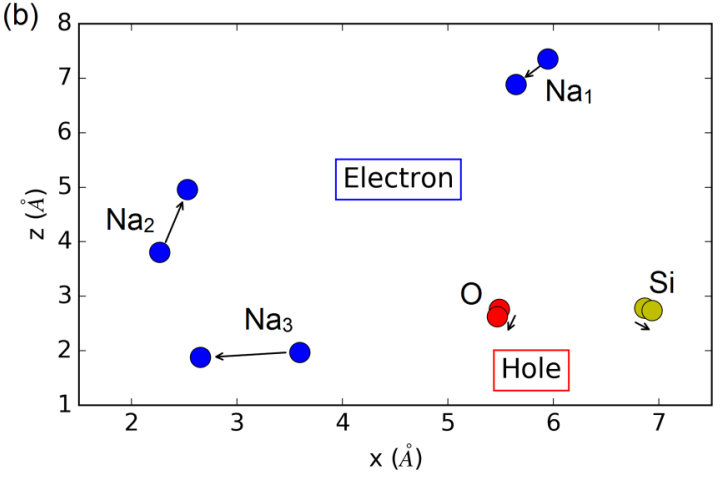

FIG. 7. Atomic displacements in the type $2 \mathrm{~L}$ center structure in the glass \#14 and electron-hole pair localization. (a) $x y$ and (b) $x z$ plane of the atomic configuration and displacement of all the relevant atoms. The arrows indicate the direction of motion from the relaxed ground state to the relaxed excited state.

One can see that three $\mathrm{Na}$ atoms have displacements of about $1.0 \AA$, which are significantly larger than the average Na displacement $(\approx 0.25 \AA)$ in this glass structure. The spin density distribution of the triplet excited state is also shown in Fig. 6. The three Na cations that experienced the largest displacements are taking part in the localization of the electron component. The individual displacements of the atomic configuration for the three $\mathrm{Na}$ cations are also highlighted in the inset. The directions of significant displacements of ions in the $L^{*}$ center with respect to the ground state in this glass model in the $x y$ and $x z$ planes are shown in Fig. 7. The difference in the interatomic distances between the relaxed ground state and the relaxed excited state for these atoms is noted in Table II.

The distance between $\mathrm{Na}_{1}$ and $\mathrm{Na}_{2}$ becomes notably shorter in the relaxed excited state (from $5.46 \AA$ to $3.74 \AA$ ), and the electron component gets localized in the close vicinity of these cations. The third sodium $\left(\mathrm{Na}_{3}\right)$, that is participating in this

TABLE II. Comparison of the interatomic distances between the atoms forming the triplet exciton in the glass structure \#14 in the relaxed ground state and the relaxed excited state.

\begin{tabular}{lcc}
\hline \hline & Interatomic distance $(\AA)$ & \\
\hline Atom pair & Ground state & Excited state \\
\hline $\mathrm{Na}_{1}-\mathrm{Na}_{2}$ & 5.46 & 3.74 \\
$\mathrm{Na}_{3}-\mathrm{O}$ & 2.23 & 3.02 \\
$\mathrm{Si}-\mathrm{O}$ & 1.57 & 1.64 \\
\hline \hline
\end{tabular}
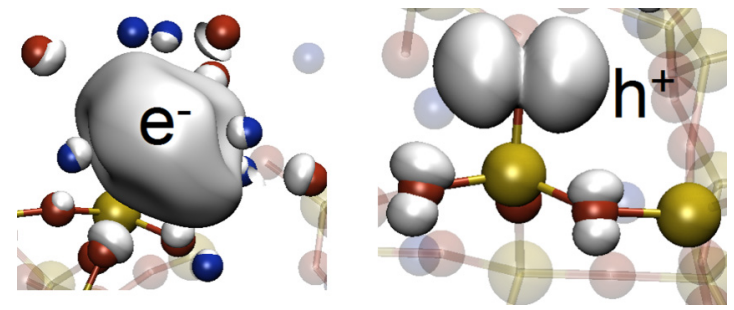

FIG. 8. Left panel: Spin density distribution of an electron trap $\mathrm{E}_{1}^{-}$. The extra electron $\left(\mathrm{e}^{-}\right)$is well localized on a cluster of $\mathrm{Na}$ ions within the glass structure. Right panel: Spin density distribution of a hole trap $\mathrm{H}_{1}^{+}$. The extra hole $\left(\mathrm{h}^{+}\right)$is well localized on a nonbridging oxygen atom. Na ions are blue, $\mathrm{Si}$ are yellow, and $\mathrm{O}$ are red. The spin density isovalue is 0.002 for both distributions.

cluster, is moving further away from the nonbridging oxygen atom on which the hole component of the exciton is well localized. We note that $3.7 \AA$ is a characteristic interatomic distance between the ions in small $\mathrm{Na}$ clusters [47], which may explain the character of this relaxation. The $\mathrm{Na}-\mathrm{O}$ bond elongates from $2.23 \AA$ to $3.02 \AA$. The Si-O bond was also stretched slightly from $1.57 \AA$ to $1.64 \AA$.

\section{E. $\mathrm{E}_{1}^{-}$and $\mathrm{H}_{1}^{+}$centers}

An extra electron was added to the relaxed ground state and the geometry of each of the systems was then re-optimized. The electronic structure of the 20 glass models exhibits a state located below the conduction band minimum (see Fig. 2). This state is the highest occupied molecular orbital mostly due to the Na ion states. The average position of this state, from the 20 glass models, is $\approx 1.2 \mathrm{eV}$ below the bottom of the conduction band and ranges from 0.8 to $1.5 \mathrm{eV}$, indicating a relatively shallow electron trap. This agrees well with Mackey's study [7], in which they assumed that the electron centers in sodium silicate glass are relatively stable at low temperatures and can be easily bleached by illuminating the glass with $1-3 \mathrm{eV}$ light.

Consequently, we could expect the extra electron to be localized on $\mathrm{Na}$ cations. The analysis of the spin density distribution, for the 20 glass structures, reveals a concept analogous to what we have seen above about the localization of the electron component in an excited $\mathrm{L}$ center state. The extra electron is localized amongst a cluster of three $\mathrm{Na}$ cations within the glass structure. An example of $\mathrm{E}_{1}^{-}$center formation in one of our glass models is shown in Fig. 8 (left panel).

A hole was added to each neutral system, and the total energy of the system was minimized with respect to its atomic coordinates. The analysis of the spin density distribution leads to similar observation with the hole component localization in an excited L center state. The hole is well localized on a single nonbridging oxygen atom and an example of $\mathrm{H}_{1}^{+}$ center formation in one of our glass models is shown in Fig. 8 (right panel).

\section{DISCUSSION AND CONCLUSIONS}

The results of this work support the model proposed in previous studies $[7,8,12,13,15]$ that the optical parameters of the $\mathrm{L}$ center are determined by the interaction of the excited electron with several sodium cations, which becomes possible 
in the presence of regions with a high local concentration of alkali cations within the glass structure. According to the modified continuous random network model [48], the network of the $\mathrm{SiO}_{4}$ units in a pure glass is significantly modified by the addition of alkali cations. The modifier cations break the bonds between the $\mathrm{Si}$ and $\mathrm{O}$ atoms, and they are linked predominantly to the $\mathrm{SiO}_{4}$ tetrahedra with weak and nondirectional bonds to nonbridging oxygen atoms. It is also well known that the alkali modifiers tend to cluster at low concentrations.

The calculated luminescence energies for type $1 \mathrm{~L}$ center structures are in good agreement with those corresponding to the maximum of the experimental luminescence spectrum, suggesting that such structures prevail in the real glass samples. Other structures give luminescence energies at the red tail of the spectrum, which has been measured experimentally down to around $2 \mathrm{eV}[17,46]$. We note that transitions with lower energies are likely to be quenched nonradiatively. Our statistics and cell size do not allow us to conclude whether there is a whole continuum of $\mathrm{Na}$ cluster structures in the glass or these are limited to types 1 and 2. Some of the more delocalized excited states contributing to the blue tail of the photoluminescence are omitted in our calculations due to cell size limitations. We also note that our results sample only the lowest triplet excitations and the corresponding relaxed exciton states and that higher excitations could also contribute to the observed luminescence spectrum.

The calculations of the individual $\mathrm{E}_{1}^{-}$and $\mathrm{H}_{1}^{+}$centers are consistent with the results from the excited states, in terms of the character of the localization of the electron and hole traps in our glass models. Our calculations agree with Mackey's model [7], which suggests that $\mathrm{x}$-ray irradiation creates electron and hole color centers in the defects present in the glass structure before irradiation. The shallow trapping levels for both electrons and holes lying, respectively, adjacent to the conduction and valence bands are clearly seen in Fig. 2. We note that these states are intrinsic to the glass structure.

The broad range of the calculated luminescence energies reflects the presence of inequivalent local atomic structures for the sodium cations within the glass structure. It has been observed that the electron localization is mostly restricted to an interstitial cavity and the vicinity of three or four neighboring sodium ions. The different kinds of local arrangements of $\mathrm{Na}$ cations that can be found in a sodium silicate glass create a spectrum of different localized states. Our calculations allow us to identify structural motifs responsible for particular luminescence energies and thus shed light on the extended L center model, which describes the localized exciton states of electronic excitations in sodium silicate glasses. The results demonstrate that, combined with the spectroscopic data, theoretical simulations represent a powerful tool for revealing the local structure of oxide glasses.

\section{ACKNOWLEDGMENTS}

K.K. was supported through the Impact Studentship scheme at UCL co-funded by the IHI Corporation and UCL. Via our membership of the UK's HEC Materials Chemistry Consortium, which is funded by EPSRC (EP/L000202), this work used the ARCHER UK National Supercomputing Service (http://www.archer.ac.uk). K.K. and A.L.S. would like to thank A.-M. El-Sayed and A. N. Trukhin for useful discussions.
[1] D. E. Day, J. Non-Cryst. Solids 21, 343 (1976).

[2] B. Mysen and P. Richet, Silicate Glasses and Melts. Properties and Structure (Elsevier, Amsterdam, 2005).

[3] N. F. Mott and E. A. Davis, Electronic Processes in Non-Crystalline Materials (Clarendon Press, Oxford, UK, 1971).

[4] D. L. Griscom, J. Non-Cryst. Solids 31, 241 (1978).

[5] L. Skuja, J. Non-Cryst. Solids 239, 16 (1998).

[6] D. Ehrt, IOP Conf. Ser.: Mater. Sci. Eng. 2, 012001 (2009).

[7] J. H. Mackey, H. L. Smith, and A. Halperin, J. Phys. Chem. Solids 27, 1759 (1966).

[8] A. N. Trukhin, M. N. Tolstoi, L. B. Glebov, and V. L. Savelev, Phys. Status Solidi B 99, 155 (1980).

[9] A. R. Kangro, M. N. Tolstoi, I. K. Vitol, V. J. Grabovski, and J. E. Kariss, Fiz. Khim. Stekla 4, 717 (1978).

[10] G. H. Siegel, J. Non-Cryst. Solids 13, 372 (1973).

[11] N. F. Mott, Contemp. Phys. 18, 225 (1977).

[12] A. N. Trukhin, L. E. Intenberg, L. B. Glebov, V. L. Savelev, and U. N. Tolstoi, Fiz. Tverd. Tela 27, 3101 (1985).

[13] A. N. Trukhin, J. Non-Cryst. Solids 189, 1 (1995).

[14] A. N. Trukhin, L. B. Glebov, and M. N. Tolstoi, Sov. Phys. Chem. Glasses 14, 547 (1988).

[15] A. N. Trukhin, J. Non-Cryst. Solids 123, 250 (1990).

[16] A. F. Zatsepin, V. B. Guseva, and D. A. Zatsepin, Glass Phys. Chem. 34, 709 (2008).
[17] D. A. Zatsepin and A. F. Zatsepin, J. Surf. Investig. 8, 726 (2014).

[18] V. I Arbuzov, A. F. Zatsepin, V. S. Kortov, M. N. Tolstoi, and V. V. Tyukov, J. Non-Cryst. Solids 134, 208 (1991).

[19] J. Du and A. N. Cormack, J. Non-Cryst. Solids 349, 66 (2004).

[20] A. Pedone, G. Malavasi, M. C. Menziani, A. N. Cormack, and U. Serge, J. Phys. Chem. B 110, 11780 (2006).

[21] A. Pedone, G. Malavasi, A. N. Cormack, U. Serge, and M. C. Menziani, Theor. Chem. Acc. 120, 557 (2008).

[22] Y. Xiang, J. Du, L. B. Skinner, C. J. Benmore, A. W. Wren, D. J. Boyd, and M. R. Towler, RSC Adv. 3, 5966 (2013).

[23] A. Pedone, G. Malavasi, M. C. Menziani, U. Serge, and A. N. Cormack, J. Phys. Chem. C 112, 11034 (2008).

[24] Y. Xiang, J. Du, M. M. Smedskjaer, and J. C. Mauro, J. Chem. Phys. 139, 044507 (2013).

[25] K. Konstantinou, P. V. Sushko, and D. M. Duffy, J. Non-Cryst. Solids 422, 57 (2015).

[26] W. Smith and T. R. Forester, J. Mol. Graph. 14, 136 (1996).

[27] P. P. Ewald, Ann. Phys. 369, 253 (1921).

[28] M. P. Allen and D. J. Tildesley, Computer Simulation of Liquids (Clarendon Press, Oxford, UK, 1989).

[29] See Supplemental Material at http://link.aps.org/supplemental/ 10.1103/PhysRevB.94.174202 for Na-O and Na-Na interatomic distances as a function of (a) the size of the glass model and 
(b) the cooling rate, pairwise radial distribution functions for $\mathrm{Na}-\mathrm{O}$ and $\mathrm{Na}-\mathrm{Na}$ interatomic distances, sodium aggregation with other sodium atoms within the glass structure, and a comparison of geometrical properties between the glass structure obtained using the classical force field and the relaxed neutral structure after the DFT optimization.

[30] A. Tilocca, J. Chem. Phys. 139, 114501 (2013).

[31] J. VandeVondele, M. Krack, F. Mohamed, M. Parrinelo, T. Chassaing, and J. Hutter, Comput. Phys. Commun. 167, 103 (2005).

[32] G. Lippert, J. Hutter, and M. Parrinello, Mol. Phys. 92, 477 (1997).

[33] J. VandeVondele and J. Hutter, J. Chem. Phys. 127, 114105 (2007).

[34] S. Goedecker, M. Teter, and J. Hutter, Phys. Rev. B 54, 1703 (1996).

[35] M. Guidon, J. Hutter, and J. VandeVondele, J. Chem. Theory Comput. 5, 3010 (2009).

[36] M. Guidon, J. Hutter, and J. VandeVondele, J. Chem. Theory Comput. 6, 2348 (2010).

[37] R. O. Jones and O. Gunnarsson, Rev. Mod. Phys. 61, 689 (1989).
[38] A. Hellman, B. Razaznejad, and B. I. Lundqvist, J. Chem. Phys. 120, 4593 (2004).

[39] A. M. El-Sayed, M. B. Watkins, A. L. Shluger, and V. V. Afanas'ev, Microelectron. Eng. 109, 68 (2013).

[40] A. M. El-Sayed, M. B. Watkins, V. V. Afanas'ev, and A. L. Shluger, Phys. Rev. B 89, 125201 (2014).

[41] X. Yuan and A. N. Cormack, J. Non-Cryst. Solids 283, 69 (2001).

[42] A. N. Cormack and J. Du, J. Non-Cryst. Solids 293-295, 283 (2001).

[43] A. C. Wright, A. G. Clare, B. Bachra, R. N. Sinclair, A. C. Hannon, and B. Vessal, Trans. ACA 27, 239 (1991).

[44] C. Mazzara, J. Jupille, A. M. Flank, and P. Lagarde, J. Phys. Chem. B 104, 3438 (2000).

[45] R. A. Murray and W. Y. Ching, J. Non-Cryst. Solids 94, 144 (1987).

[46] A. N. Trukhin, in International School of Solid State Physics, 17th course, NATO Science Series. II Mathematics, Physics and Chemistry, edited by D. Griscom, G. Pacchioni, and L. Skuja (Springer, New York, 2000), pp. 235-283.

[47] G. Pal, G. Lefkidis, H. C. Schneider, and W. Hubner, J. Chem. Phys. 133, 154309 (2010).

[48] W. H. Zachariasen, J. Am. Chem. Soc. 54, 3841 (1932). 